\title{
Coulisses
}

Revue de théâtre

25 | Hiver 2002

Varia

\section{Philippe Minyana rencontre les apprentis acteurs et/ou metteurs en scène de Inventaires du Lycée Ledoux}

\section{Gilles Scaringi}

\section{(2) OpenEdition}

Journals

Édition électronique

URL : http://journals.openedition.org/coulisses/5988

DOI : 10.4000/coulisses.5988

ISSN : 2546-9460

Éditeur

Presses universitaires de Franche-Comté

Édition imprimée

Date de publication : 1 janvier 2002

Pagination : 56-61

ISBN : 2-84627-052-X

ISSN : $1150-594 X$

Référence électronique

Gilles Scaringi, «Philippe Minyana rencontre les apprentis acteurs et/ou metteurs en scène de Inventaires du Lycée Ledoux 》, Coulisses [En ligne], 25 | Hiver 2002, mis en ligne le 24 octobre 2019, consulté le 15 novembre 2019. URL : http://journals.openedition.org/coulisses/5988; DOI : 10.4000/ coulisses.5988

Ce document a été généré automatiquement le 15 novembre 2019.

Coulisses 


\title{
Philippe Minyana rencontre les apprentis acteurs et/ou metteurs en scène de Inventaires du Lycée Ledoux
}

\author{
Gilles Scaringi
}

1 La rencontre entre un auteur et une classe est toujours un événement exceptionnel mais un exercice délicat, à plus forte raison lorsque l'intervenant est Philippe Minyana et l'auditoire, les élèves d'une option théâtre, qui se collent à son texte le plus joué : Inventaires ${ }^{1}$. L'exercice devient plus difficile encore lorsqu'il s'agit de commenter avec lui sa dernière création, Pièces, accueillie cet hiver au Nouveau Théâtre dans la mise en scène de Robert Cantarella. Néanmoins, pour ces lycéens, échanger avec un auteur dramatique reconnu est la preuve que le théâtre, quelle que soit la manière dont on le pratique, est bien vivant.

\section{Besançon, un pèlerinage}

2 L'Auditorium du lycée est situé au dernier étage de la tour dont la vue imprenable sur Besançon plaît aussitôt à Philippe Minyana. Il en profite pour rappeler qu'il est né ici, qu'il y a fait ses études universitaires et que c'est ici, avec Jacques Vingler, qu'il s'est initié à l'art dramatique. D'emblée, il nous confie quel choc cela a été pour lui de revenir dans sa ville natale après une très longue absence : les quais, la place Granvelle, le théatre municipal, la faculté des lettres. Tout lui a parlé violemment. Mais ce pèlerinage lui est nécessaire, non parce qu'on le joue au Nouveau Théâtre et que le déplacement est bien sûr professionnel, mais parce que c'est un retour à une période charnière de sa vie. Il s'attarde quelques instants sur ses années de formation, évoque son métier d'enseignant qu'il a exercé pendant dix ans dans les Vosges, se remémore l'écriture de ses tout premiers textes puis relate son départ définitif pour Paris à l'âge de 33 ans. Départ, mais rupture avec son passé, avec sa géographie sentimentale, et surtout avec ce fragment de sa vie, intime, douloureux, salvateur tout à la fois, et dont on retrouve de nombreux échos dans ses pièces majeures. 
Minyana n'insiste pas. Mais on comprend que la décision fut irrévocable. Écrire, simplement, toujours, sans faire la moindre concession à la facilité et aux modes. L'immersion dans la vie intellectuelle et artistique parisienne lui fournit l'occasion de rencontres déterminantes: Lucien Attoun à "Théâtre ouvert", Édith Scob, Alain Françon, Robert Cantarella avec lequel il se lie d'amitié à l'école d'Antoine Vitez. Sa première pièce éditée, Fin d'été à Baccarat, malgré une dramaturgie classique, préfigure l'audace des écrits ultérieurs et restitue assez ses préoccupations de l'époque : prendre pied dans la réalité quotidienne ici le milieu enseignant croisant le fait divers pour la transformer avec cette teinte d'humour et de dérision qui n'appartient qu'à lui. Minyana rappelle aux élèves que la quête de la forme constitue la charpente de son travail, déjà perceptible dans ce premier texte publié.

\section{Le travail de l'écrivain}

Le contact avec eux est direct, précis, chaleureux. Il répond avec la même générosité et la même franchise à chacune des questions. Comment devient-on auteur de théâtre? Quelle est sa démarche artistique? Qu'est-ce que le théâtre pour lui? Minyana dissimule mal son bonheur d'accoucher d'un texte, même quand celui-ci est une commande. L'inspiration ne vient pas tout de suite, prévient-il. D'abord il faut s'imprégner de la matière sur laquelle il va écrire, durant plusieurs mois parfois, et lire tout ce qui se présente à lui : la presse, la littérature légère sans oublier les maîtres comme Dostoïewski ou Cioran, dont la fréquentation lui est roborative. Car il a besoin de cette lucidité noire que l'auteur des Frères Karamazov ou celui des Cahiers jette sur le monde qui nous entoure pour éclairer son propre désenchantement, pour s'en nourrir. Et pour s'en nourrir. Et pour mieux l'appréhender aussi. Cette lente gestation implique une disponibilité d'esprit absolue, une errance totale au milieu de ses objets familiers. Une fois ce travail intérieur achevé, la forme trouvée, l'écriture peut alors commencer.

Pour écrire, Minyana ne recourt ni au traitement de texte ni à la machine à écrire. Il lui faut la plume et le papier, afin de reprendre, souligner, raturer, coller, recommencer. Il a besoin de voir les petits signes noirs que la main s'applique à dessiner sur la feuille tachée et froissée. Plus tard seulement, le texte sera dactylographié. Il n'a de cesse de le répéter: non, l'écriture n'est ni contrainte ni souffrance. Elle est un aboutissement normal du processus de la création. Elle devient parole à entendre, à jouer, à voir.

6 Très vite, l'auditoire comprend mieux ce qu'il traque depuis toujours : l'épisode ténu qui fait basculer l'individu dans le fait tragiquement dérisoire. Et seule la parole de théâtre, monologuée ou fragmentée, lui permet d'en restituer toute la profondeur. L'absence de ponctuation, le flux des mots, le rythme et la respiration des phrases, l'opacité de la typographie sont les marques signifiantes de cette démarche originale, de cette langue réinventée à partir de la parole des autres comme on le constate dans ses Drames brefs, dans Chambres, dans Inventaires. Se pose alors la question de la mise en forme théâtralisée.

\section{Le rapport à la mise en scène}

7 Là, les élèves l'interrogent sur son rapport au metteur en scène. Pour Minyana, il y a deux créations conjointes : la sienne et celle du metteur en scène. Elles n'interfèrent 
pas. Une fois le texte écrit, il est ce qu'en fera ce dernier qui suit sa propre esthétique en bâtissant son propre univers. Que ce soit pour Où vas-tu, Jérémie ? montée par Édith Scob ou Pièces par Robert Cantarella, l'auteur est resté très éloigné de la fabrication du spectacle, même si l'œuvre de Boltanski, chère à Minyana, est omniprésente dans la scénographie d'Édith Scob et si Pièces est dédiée à Robert Cantarella. On pourrait dire, pour simplifier, que se crée naturellement une osmose entre lui et le metteur en scène. L'un et l'autre n'utilisent pas les mêmes mots mais ils parlent la même langue. Ils peuvent s'entretenir du sens à donner à tel ou tel détail du texte. L'auteur s'interdira tout monopole d'interprétation. Peut-être faut-il voir là une sorte de ligne de partage : l'auteur envisage le texte imprimé dans sa forme définitive, le metteur en scène se réserve des champs de possibles dont seul le spectateur sera juge.

\section{Acteurs et metteurs en scène de Inventaires en classe de $1^{\text {re }}$}

8 Si la question est pertinente dans ce type de relation, l'est-elle pour des élèves qui auront à jouer le texte d'un auteur en face duquel ils se retrouvent tout à coup? Les questions fusent, leur angoisse se lit sur les visages : comment devront-ils interpréter les personnages d'Inventaires, Jacqueline, Angèle, Barbara sans le trahir, lui ? Comment mettre en espace ce qui pourrait être une parodie de confession publique relayée par ces trois femmes? Et les garçons de la troupe, peuvent-ils jouer des rôles féminins? L'échange plaît manifestement à Philippe Minyana qui rassure tout le monde par des conseils inspirés. Mais là non plus, il n'y a pas de règle. Tout est envisageable quant à la forme choisie. L'idée de diviser les rôles entre filles et garçons pour que chacun puisse jouer est non seulement souhaitable mais riche. Ce sera l'occasion de mettre en voix la parole des femmes avec la personnalité de chacun des comédiens. Le monologue deviendra un chœur au sens antique du terme, une polyphonie susceptible de faire entendre tous les épisodes d'une vie racontée spontanément. Pour y parvenir, Minyana préconise la quête du petit détail, l'intention inavouée qu'il faut déceler dans un passage insignifiant de la confession pour tendre ensuite toute la parole sur ce point-là. Dès lors, le texte peut offrir de nombreuses possibilités de jeu tout en sauvegardant l'esprit de la pièce : la joie de se raconter avec une dose estimable d'impudeur.

\section{Le jeu de l'acteur}

Pour jouer juste, recommande-t-il aux élèves, « il ne faut pas jouer quelque chose qui ne vous appartient pas, ne pas faire une composition, quelle qu'elle soit! Vous devez rester vous-mêmes pour transporter le texte. Le rôle du comédien, comme le dit Marguerite Duras, est d'être un passeur. Il faut faire entendre le texte. On doit considérer celui-ci comme une partition non ponctuée pour garder l'irruption de la parole en permanence, sa fluidité, son allégresse. Car ces femmes sont contentes de raconter leur vie, même s'il $y$ a des choses noires! Comme vous êtes jeunes, il ne faut pas chercher à composer des silhouettes âgées, parce que, là, vous ne feriez plus du théâtre mais du patronnage! Il faut que vous conserviez le plaisir de dire des aveux intimes avec ce que vous êtes, vous, avec votre volume, votre voix comme des chanteurs le feraient en suivant une partition. Souvent, je dis que les acteurs sont des sportifs de haut niveau. Ils concentrent tous les muscles nécessaires au moment de la performance. Pour le parleur c'est la même chose, 
il faut concentrer un corps vertical, une voix claire, un visage souriant, ouvert, puisque ça leur fait tellement plaisir à ces femmes de raconter leur vie !»

\section{Inventaires}

10 Minyana finit par énumérer les raisons pour lesquelles il a écrit Inventaires, quel pari amusant avec Édith Scob et Judith Magre est à l'origine de ce texte, comment il a recueilli les aveux de femmes qu'il a lui-même interrogées, comment la pièce a été jouée dans des endroits très différents les uns des autres, dans des mises en scène très " culottées » et comment le film de Jacques Renard a été tourné dans un hypermarché, à l'insu des consommateurs. Avant que cela ne devienne un genre prisé des fidèles du "reality show» que nous connaissons aujourd'hui, l'aveu public, le déballage cathodique, le « marathon de la parole » se trouvaient déjà dans Inventaires.

\section{Pièces}

11 Enfin, la dernière partie de la rencontre est consacrée à Pièces, qui est l'événement théâtral de la semaine à Besançon. Philippe Minyana ne demande pas aux élèves s'ils ont apprécié le spectacle, mais il leur explique pourquoi et comment il a écrit ce texte, dont la composition en neuf morceaux constitue la nouveauté. Le titre polysémique suggère cet éclatement des formes, mais également le lieu, l'habitat, les objets qui le remplissent. La trame en est l'errance, la solitude crépusculaire d'un homme, Tac, dont la fin approche et qui vient d'être délogé arbitrairement. De nombreuses figures littéraires hantent les personnages. On pense à Dostoïewski, à Beckett, à Cioran auquel Minyana a emprunté plusieurs aphorismes signalés dans le texte imprimé. L'importance des didascalies souligne le vœu de l'auteur d'être joué au plus près de l'intention dramaturgique: suivre un destin individuel dans les affres de la confrontation aux autres: voisins, artisans et commerçants du quartier où il vit, badauds mais aussi scènes de villages et scènes d'intimité familiale où cancans et rumeurs reposent sur des clichés de la conversation courante, sur le comique de répétition, sur la polyphonie mortifère de voix qui appellent et se taisent, sur l'apparition de silhouettes presque fantomatiques, sur l'émergence soudaine du fait divers qui prend une tournure shakespearienne, sur la reconstitution d'un intérieur courtelinesque brutalement schizophrène.

12 La mise en scène de Robert Cantarella sert habilement l'écriture de Minyana. Ce que le texte laisse entendre, la mise en scène le met en évidence par des trouvailles étonnantes : la démolition de tout un pan du décor ouvre soudain sur une sorte de promenade campagnarde, à la beauté lunaire, où la vie et la mort se côtoient. L'utilisation du son et de l'image donne un écho saisissant à des personnages dont la parole se trouve artificiellement décalée. Leur expression fait songer à de pâles histrions dont l'image en surimpression se mue en un film projeté sur fond de scène. Ainsi les objets familiers de Tac nous apparaissent-ils essentiels, inexorablement liés à son destin. Sans jamais tomber dans le pathos ni les effets grandiloquents, le personnage semblant sorti d'outre-tombe ressurgit dans le local d'un parti politique dont on ne sait plus s'il est l'antichambre d'une agence mafieuse ou la métaphore de son enfer intime. Enfin, l'emploi de comédiens amateurs réussit à contrebalancer le jeu 
très abouti d'acteurs confirmés, de sorte que les «habitants qui parlent » le font avec l'hésitation et l'inconfort qu'on devine. Mélange d'émotion et d'humour garanti !

Peut-être est-ce tout cela qui a troublé les lycéens, surpris par un théâtre aussi novateur. Une question d'une jeune fille a aussitôt fait réagir Minyana : «Après avoir vu votre pièce, je suis sortie du théâtre, déprimée! » En pièces, aurait-elle pu ajouter ! La discussion est salutaire. Les élèves ont besoin de comprendre ce qu'on leur montre. Pour Minyana, c'est le traitement d'un sujet grave par la dérision qui importe. Or, la salle a parfaitement réagi. Si la mise en scène avait insisté sur la solitude tragique de Tac, alors le pessimisme eût submergé les spectateurs. C'est le contraire qui s'est produit. Le comique de geste et de répétition, le saugrenu du jeu et les jeux de mouvement dans certaines situations, les joutes verbales et les jeux de mots dans d'autres, ont eu bien souvent un effet jubilatoire sur le public. Minyana se défend d'avoir voulu écrire un théatre triste, mais le thème de l'errance et de l'exil lui tient à cœur : qu'il soit urbain ou géopolitique, l'exil est un fait de notre époque. Les SDF, le quart-monde, les populations déplacées en Afrique et en Asie centrale, les sans-papiers, les réfugiés qui se morfondent dans des camps de transit, sont pour lui un phénomène très symptomatique d'une civilisation qui rejette aux frontières une partie non négligeable de ses habitants. Sans doute est-ce de cela aussi que parle Pièces. Les objets qu'on emporte avec soi deviennent en effet dérisoires: "le repose-pied, le spot, le guidon, la table pliante, les crapauds, la patère... et le meuble d'angle !» Mais le théâtre nous autorise à en rire. Là est la différence.

Cet échange aura permis aux élèves du lycée Ledoux de prendre toute la mesure de cette rencontre. À présent, ils reliront Pièces et le théâtre de Philippe Minyana autrement. Plus important encore pour eux, ils joueront Inventaires ${ }^{2}$ au mois de mai, en sachant que derrière l'écriture bouleversante de ces confessions joyeusement impudiques, se cache un écrivain sensible, fin et soucieux de rendre la parole à ceux qui l'ont perdue. Toute son œuvre est là, traversée de ces silences têtus, de ces ombres fugaces, de ces destins entrecroisés au détour d'une rue ou d'une phrase, de ces brisures secrètes que dissimule, curieusement, une parole monolithique et dont se prive le lecteur pressé ou le spectateur distrait.

\section{NOTES}

1. Avant Scène, $\mathrm{n}^{\circ} 809$, réédition dans une version différente, Éditions Théâtrales, 1993.

2. Coulisses $n^{\circ} 26$ publiera le compte rendu de notre travail sur Inventaires. 


\section{AUTEUR}

\section{GILLES SCARINGI}

Chargé de l'enseignement du théâtre au lycée Ledoux 